'Escuela de Medicina. Universidad de Valparaíso. Valparaíso, Chile. ${ }^{2}$ Escuela de Medicina. UNAB. Viña del Mar, Chile.

${ }^{3}$ Unidad de Trastornos del Ánimo. Hospital Psiquiátrico del Salvador de Valparaíso. Valparaíso, Chile.

${ }^{4}$ Escuela de Psicología, Facultad de Ciencias Sociales. Universidad de Valparaíso. Chile ${ }^{5}$ MIDAP.

${ }^{6}$ Universidad del Desarrollo, Centro de Epidemiología y Políticas de Salud, Facultad de Medicina Clínica Alemana. Santiago, Chile. ${ }^{7}$ Facultad de Medicina. Universidad de Chile. Santiago, Chile.

${ }^{8}$ Instituto de Fisiología, Facultad Ciencias, Universidad Valparaíso. Valparaíso, Chile ${ }^{9}$ Centro Interdisciplinario de Neurociencia de Valparaíso CINV. Valparaíso, Chile. ${ }^{10}$ Hospital Dr. Gustavo Fricke. Departamento de Salud Mental. Viña del Mar, Chile. "Programa de Doctorado en Psicoterapia. Pontificia Universidad Católica de Chile. Santiago, Chile.

${ }^{\mathrm{a}}$ Doctor ${ }^{\oplus}$ Psicoterapia. Pontificia Universidad Católica de Chile. Santiago. bPsicóloga. 'PhD.

¿Kinesiólogo. Magister en Salud Pública. eng. en Biotecnología.

Proyecto financiado por Fondo Nacional de Desarrollo de la Ciencia y Tecnología,

FONDECYT No 1150166 y al Fondo de Innovación para la Competitividad (FIC) del Ministerio de Economía, Fomento y Turismo, a través de la Iniciativa Científica Milenio, Proyecto IS130005. CONICYT/ Doctorado Nacional/ 2016- 21161312.

Autores declaran no tener conflictos de interés. Recibido el 10 de octubre de 2019, aceptado el 27 de marzo de 2020.

Correspondencia a: Dr. Ulises Ríos

12 norte 785 Oficina 1201. Viña del Mar, Chile. ulisesrios@gmail.com

\section{El maltrato infantil y su rol en el curso clínico de pacientes con trastorno bipolar}

\author{
ULISES RÍOS $1,2,3,5,11, \mathrm{a}$, PABLO R. MOYA ${ }^{8,9, \mathrm{c}}$, \\ ÓSCAR URREJOLA ${ }^{6, d}$, JIMENA HERMOSILLA ${ }^{1,3}$, \\ RENÉ GONZALEZ ${ }^{8, e}$, PAULINA MUÑOZ ${ }^{10}$, JAVIER MORAN ${ }^{4,5, \mathrm{c}}$, \\ PAULA SOLERVICENS ${ }^{4, b}$, JUAN PABLO JIMÉNEZ ${ }^{7,5}$
}

\section{History of child abuse among patients with bipolar disorders}

Background: A history of child abuse is common and has a significant impact in the clinical course of patients diagnosed with bipolar disorders (BD). Aims: To assess the frequency of child abuse experiences in patients BD type I and to evaluate its association with clinical course and cognitive functioning variables. Material and Methods: 117 patients with BD aged $45 \pm 14$ years (66\% women) answered the Childhood Trauma Questionnaire (CTQ). The clinical course (illness onset, history of suicide attempts and number of hospitalizations) was obtained from medical records. Cognitive functioning was evaluated through social and non-social cognition tasks. Results: 64\% of participants reported some type of child abuse. This variable was associated with an early onset of the disease (Odds ratio $(O R)=3.3 ; p<0.02)$, increased risk of suicide attempts $(O R=2.4$; $p<0.04)$ and specific disturbances in social cognitive tasks. Conclusions: Our study supports evidence of a common history of child abuse in patients with $B D$. Although child abuse predicts a worse clinical course, major clinical practice guidelines, as well as research designs, do not highlight this evidence.

(Rev Med Chile 2020; 148: 204-210)

Key words: Bipolar Disorder; Child Abuse; Cognition; Psychological Trauma; Suicide, Attempted.
$\mathrm{L}$ a omisión de los antecedentes de estrés temprano en pacientes con enfermedades psiquiátricas caracterizadas por síntomas psicóticos parece ser un hecho de lamentable frecuencia en la práctica clínica. Pese a que los servicios de salud mental están respondiendo cada vez de mejor forma a las necesidades clínicas que emergen en víctimas de maltrato infantil, las personas que han padecido cuadros clínicos con síntomas psicóticos parecen ser la excepción, evi- denciando importantes índices de subdiagnóstico y consecuente ausencia de acceso a tratamiento ${ }^{1}$.

La Organización Mundial de la Salud estima que un cuarto de la población adulta reporta haber sido abusada físicamente durante su infancia, siendo víctima específicamente de abuso sexual, una de cada 5 mujeres, y uno de cada 13 hombres $^{2}$. Las cifras suelen ser aún mayores cuando las mediciones se han realizado en pacientes con Trastornos psiquiátricos severos, como Esqui- 
zofrenia y Trastorno Bipolar (TB), constatando frecuencias de maltrato infantil (MI) hasta tres veces más altas.

Una de las investigaciones que ha estudiado el antecedente de MI en pacientes con TB encontró cifras cercanas a $51 \%{ }^{4}$, reportando además que la frecuencia de los subtipos de MI alcanza el 37\% para abuso emocional, $24 \%$ abuso físico, $21 \%$ abuso sexual, $24 \%$ negligencia emocional y $12 \%$ negligencia física. Los autores reportaron además que un tercio de los pacientes bipolares presentó una combinación de los diferentes subtipos de trauma ${ }^{4}$.

Varios estudios han relacionado el antecedente de MI con un curso clínico de peor pronóstico en pacientes con TB. Una revisión sistemática publicada en el año 2011, encontró que el antecedente de trauma infantil se relacionó con un inicio más temprano de la enfermedad, mayor presencia de conductas suicidas y abuso de sustancias 5 . Un reciente meta-análisis confirmó lo anterior, constatando además la asociación con un curso clínico caracterizado por un mayor número de episodios anímicos, síntomas de mayor severidad y mayor co-morbilidad psiquiátrica ${ }^{6}$. Adicionalmente se han encontrado asociaciones entre MI y una peor respuesta farmacológica a tratamiento con $\mathrm{Litio}^{7}$; mayor retraso diagnóstico ${ }^{8}$ y una modificación del tipo de síntomas prodrómicos de la enfermedad bipolar?.

La identificación del trauma infantil como un potencial modificador del curso clínico en el TB parece bien documentada, sin embargo, su incorporación en guías de práctica clínica tanto nacionales como internacionales ha sido mayormente omitida. Lo mismo ha ocurrido en algunas revisiones sistemáticas y meta-análisis que intentan describir las características psicopatológicas del TB. Un ejemplo de esto último, son los resultados reportados en artículos de revisión acerca del grado de disfunción cognitiva en pacientes con $\mathrm{TB}^{10-12}$, los cuales han omitido la incorporación del estrés temprano en el análisis de sus resultados y conclusiones. Desde la evidencia disponible sobre el impacto del estrés temprano en el funcionamiento cognitivo ${ }^{13-15}$ y la mayor frecuencia de MI reportada en pacientes bipolares, la desatención de dicho antecedente en el estudio cognitivo de pacientes con TB podría estar generando conclusiones apresuradas sobre las características psicopatológicas de la enfermedad.
Impresiona que tanto en el ámbito clínico, como en la mayoría de la investigación, existe una aproximación de marcado sesgo biomédico en la comprensión de una enfermedad de conocido componente genético como el TB. El presente estudio tiene por objetivo medir la frecuencia del antecedente de MI en pacientes con TB tipo I en eutimia, evaluando la asociación con variables de curso clínico y funcionamiento cognitivo. La evidencia antes reportada, y la ausencia de datos nacionales sobre la frecuencia y rol del antecedente de MI en este específico grupo clínico, fundamentan la relevancia ético-clínica de nuestra pregunta de investigación.

\section{Material y método}

\section{Participantes}

Muestra no probabilística de 122 pacientes, entre 18 y 65 años, con diagnóstico de TB tipo I, según criterios DSM-IV TR pertenecientes a la Unidad de Trastornos del Ánimo del Hospital Psiquiátrico del Salvador de Valparaíso y Unidad de Psiquiatría ambulatoria del Hospital Gustavo Fricke de Viña del Mar.

\section{Diseño}

Estudio analítico observacional de corte transversal para la medición de la frecuencia del antecedente de MI y su asociación con variables de curso clínico en pacientes con TB tipo I en eutimia

\section{Procedimiento e instrumentos}

Se ingresaron a protocolo pacientes con TB tipo I en eutimia, constatada a través de la aplicación de escala Hamilton-17 para sintomatología depresiva y Young Mania Rating Scale (YMRS) para sintomatología maniaca. Se excluyeron pacientes con consumo activo de drogas y antecedentes de terapia electro-convulsiva durante los últimos 3 meses.

El antecedente de MI fue evaluado mediante Childhood Trauma Questionnaire Short-Form ${ }^{16,17}$, en su versión en español. Este instrumento de auto-reporte consta de 28 ítems que indagan sobre 5 diferentes subtipos de MI: abuso sexual, abuso emocional, abuso físico, negligencia física y negligencia emocional. Debido a razones ético-clínicas, el instrumento de auto-reporte fue respondido por el paciente en un encuadre clínico, confidencial, y en presencia de su médico tratante. 
El médico tratante fue el encargado de recopilar antecedentes clínico-asistenciales mediante entrevista y análisis de ficha clínica. Se indagó sobre la edad de inicio de la enfermedad bipolar, conceptualizada como primer episodio anímico; número de hospitalizaciones y número de intentos de suicidio. El funcionamiento cognitivo fue evaluado mediante pruebas de cognición social y no social. Para la primera se utilizó TASIT (The Awareness of Social Inference Test $)^{18}$ en español, prueba que evalúa la capacidad de reconocer emociones mediante la presentación de 20 micro-videos en los cuales actores simulan situaciones que representan emociones de miedo, asco, sorpresa, tristeza y enojo. Adicionalmente se utilizó el Interpersonal Reactivity Index en españo ${ }^{19}$ para medir un tipo específico de respuesta empática ("empathic distress") consistente en la capacidad de compartir el estado emocional de otro, pero con un importante grado de ansiedad y malestar "auto-orientado". Otra subescala del mismo instrumento permitió la medición del grado de "respuesta compasiva", en la cual el proceso empático se encuentra acompañado de sentimientos de afecto y/o preocupación por el estado emocional del otro, con la presencia adicional de un deseo por aliviar el padecer del otro ${ }^{20}$. Las medidas de cognición no-social evaluaron los dominios de orientación y atención, memoria, fluencia verbal, lenguaje y habilidades visuo-espaciales, mediante el instrumento ACER-versión chilena ${ }^{21}$.
El estudio fue aprobado por el comité de ética de la Dirección del Servicio de Salud Valparaíso-San Antonio. Todos los pacientes completaron el proceso de consentimiento informado antes de ingresar al estudio.

\section{Análisis estadístico}

Los datos fueron analizados mediante software Stata 14.0, se presentan como frecuencias en la fase descriptiva. Para el análisis se colapsaron las categorías de abusos para dicotomizar las respuestas según puntos de corte basados en la literatura. Se utilizó regresión logística para determinar Odds Ratio e intervalos de confianza del 95\%. Además, se utilizó Rho de Spearman para correlacionar MI con cognición social. Para todo el análisis de determinó el nivel de confianza menor a 0,05.

\section{Resultados}

\section{Frecuencia de maltrato infantil}

La muestra presentó una edad promedio de 45,5 años (13,9 DS), siendo $65,7 \%$ de sexo femenino. El 64\% de los pacientes reportó haber sido víctima de al menos un tipo de maltrato durante su infancia. La frecuencia según el subtipo de MI fue de $42 \%$ para abuso sexual; $30 \%$ abuso físico; $23 \%$ abuso emocional; $17 \%$ negligencia emocional y 14\% negligencia física (Figura 1). En relación a la intensidad del abuso infantil, 10\% de los pacien-

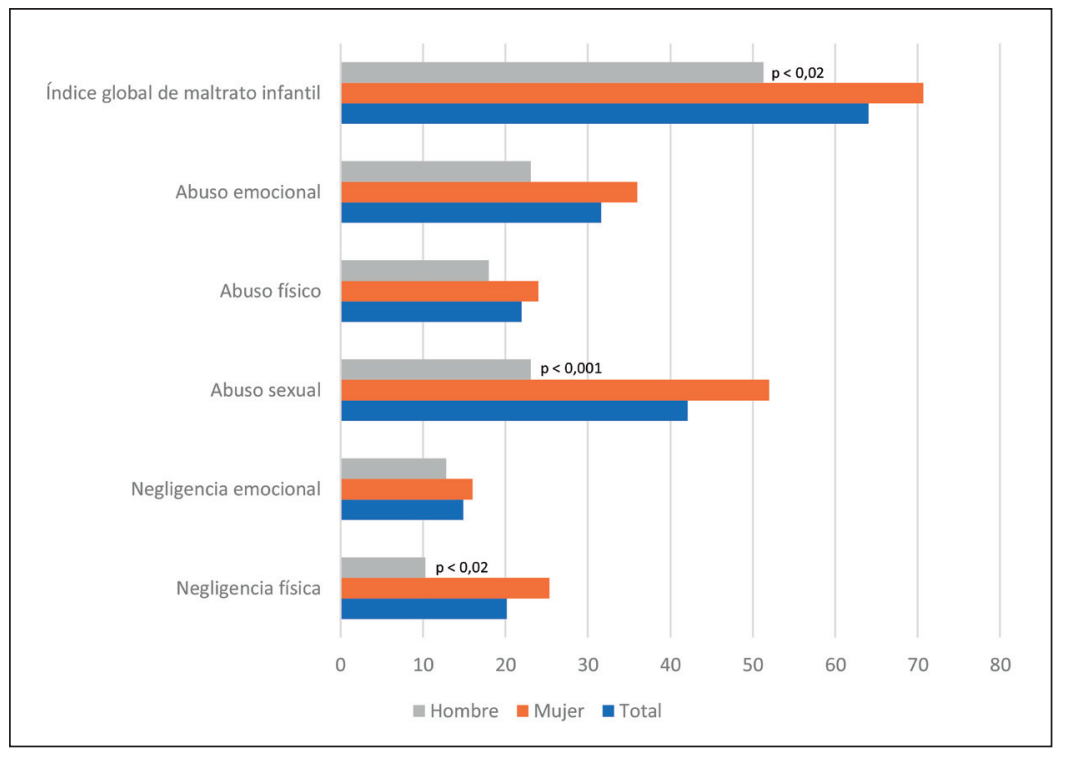

Figura 1. Frecuencia de subtipos de maltrato infantil en pacientes con Trastorno Bipolar tipo I, según sexo. Se presentan las frecuencias de maltrato infantil según subtipos. Cada variable se grafica de forma diferenciada para hombres, mujeres y muestra general. Los valores $p$ hacen referencia a la diferencia entre hombres y mujeres. 
tes fueron víctimas de los tres subtipos de abuso (sexual, emocional y físico) y $17 \%$ fue víctima de al menos 2 subtipos de abuso. El análisis por sexo dio cuenta que $71 \%$ de las mujeres y $51 \%$ de los hombres habían sido víctimas de algún tipo de MI. Dicha diferencia fue estadísticamente significativa $(\mathrm{p}<0,02)$.

\section{Maltrato infantil y variables clínico-asistenciales}

Nuestro estudio demuestra que pacientes bipolares tipo I con antecedentes de MI presentan un peor curso clínico en comparación con pacientes con el mismo diagnóstico, pero sin historia de trauma temprano. En el análisis sobre la edad de inicio de la enfermedad, conceptualizada como la edad de presentación del primer episodio anímico, ya sea depresivo o maníaco, el estudio demostró una asociación estadísticamente significativa entre el antecedente de MI y un inicio de enfermedad a una edad más temprana. En aquellos pacientes que iniciaron la enfermedad antes de los 20 años, el riesgo de haber presentado algún tipo de maltrato durante la infancia fue 3,3 veces mayor (OR: 3,3; IC $[1,2-9,2], p<0,02)$, encontrando además una asociación con el subtipo abuso emocional (OR: $2,7[1,09-6,9] \mathrm{p}<0,03)$ y una tendencia en el límite de la significancia estadística con el subtipo abuso sexual (OR: 2,2 [0,97-5,3] p < 0,058). En el caso específico del antecedente de abuso sexual y su asociación con un inicio de la enfermedad antes de los 19 años, se constató un OR de 2,4 (IC [1,02-
5,8]. $\mathrm{p}<0,04)$. En la Figura 2, se muestran las respectivas medidas de asociación entre el inicio de la enfermedad bipolar antes de los 20 años y el antecedente de MI como medida total, y como subtipos específicos de maltrato.

Dado el alto riesgo suicida en pacientes bipolares reportado en la literatura, nuestra investigación evaluó la asociación entre el MI y el antecedente clínico de intentos de suicidio. Nuestros datos confirman que el antecedente de MI se asoció de forma significativa con los antecedentes clínicos de suicidalidad dentro de la muestra estudiada. En el grupo de pacientes que han presentado al menos un intento de suicidio, el riesgo de haber sido maltratados en la infancia fue 2,4 veces mayor $(\mathrm{OR}=2,4 ; \mathrm{IC}[1,01-5,76] \cdot \mathrm{p}<0,04)$. El riesgo fue aún mayor cuando se analizó según subtipo de maltrato, encontrando un $\mathrm{OR}=3,5$ (IC $[1,45-8,6]$ $\mathrm{p}<0,005)$ entre el antecedente de abuso sexual y el antecedente clínico de al menos un intento de suicidio. La Figura 3, muestra las medidas de asociación entre el antecedente de al menos un intento de suicidio y las diferentes medidas de MI. Finalmente, nuestro estudio no encontró asociaciones entre el número de hospitalizaciones y el antecedente de trauma temprano.

\section{Maltrato infantil y funcionamiento cognitivo}

En la medición de índices de cognición social, específicamente el rendimiento en una tarea de reconocimiento de emociones, los pacientes con

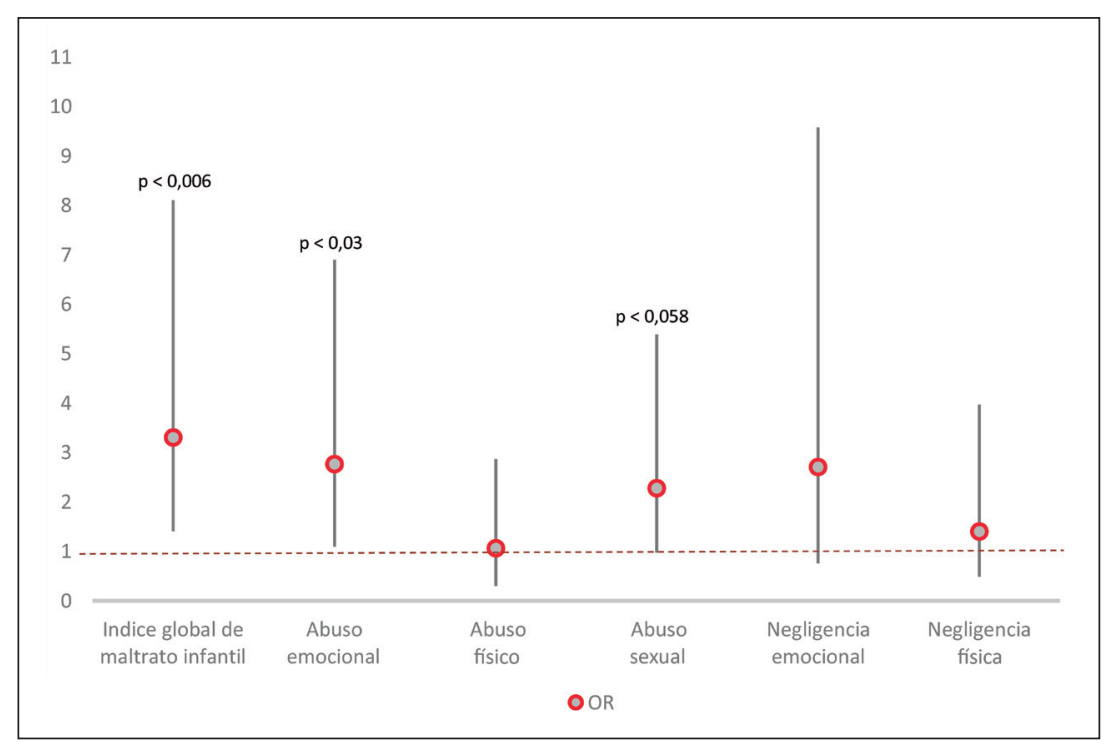

Figura 2. Asociación entre inicio temprano de la enfermedad (antes de los 20 años) y subtipos de maltrato infantil en pacientes con Trastorno Bipolar tipo I. La figura grafica los valores de Odds ratio (OR) e intervalos de confianza 95\%, entre los diferentes sub-tipos de maltrato infantil y el inicio de la enfermedad bipolar antes de los 20 años. 


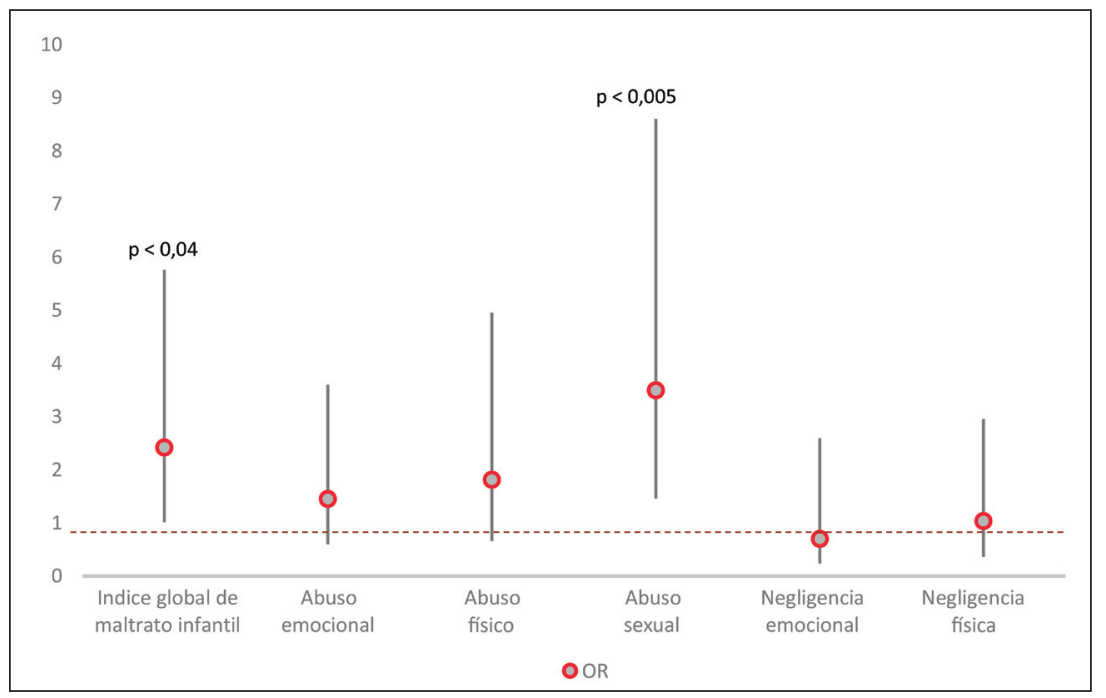

Figura 3. Asociación entre maltrato infantil y antecedente de intentos de suicidio en pacientes con Trastorno Bipolar tipo I. La figura grafica los valores de Odds ratio (OR) e intervalos de confianza 95\%, entre los diferentes sub-tipos de maltrato infantil y el antecedente de intento de suicidio.
TB que habían sido víctimas de abuso emocional reconocieron más situaciones de "enojo" (rho spearman 0,$3 ; \mathrm{p}<0,03)$ en comparación con pacientes sin antecedentes de trauma temprano. En el grupo de pacientes con historia de abuso físico, hubo mayor reconocimiento de la emoción "tristeza" (rho spearman 0,3; p < 0,01) en relación a pacientes bipolares sin antecedentes de abuso. En los dominios de la ruta afectiva de la cognición social, se encontró que a mayor intensidad de maltrato infantil menor fue el índice de "respuesta compasiva", una tendencia observada sólo en pacientes menores de 50 años $(\mathrm{p}<0,02)$. Nuestros resultados no encontraron diferencias significativas en medidas de "empathic distress" entre ambos grupos.

La comparación del funcionamiento cognitivo entre pacientes bipolares con y sin antecedentes de MI no evidenció diferencias significativas en medidas neuro-cognitivas (cognición no-social) como orientación y atención, memoria, fluencia verbal, lenguaje y habilidades viso-espaciales.

\section{Discusión}

Nuestra investigación ha constatado una alta frecuencia de MI en pacientes con TB tipo I en eutimia, encontrando además que dicho antecedente se asocia con una edad más temprana de inicio de la enfermedad, un mayor riesgo de intentos de suicidio y alteraciones específicas en el rendimiento en tareas de cognición social. Los hallazgos aquí reportados son coincidentes con la evidencia disponible ${ }^{6,22}$, enfatizando la asociación entre el trauma temprano y un peor curso clínico en el TB.

Nuestro estudio es pionero a nivel nacional en medir el antecedente de MI en pacientes con TB. Dada la ausencia de datos previos en este contexto clínico, la magnitud de la frecuencia encontrada solo es posible de dimensionar en relación a índices de MI constatados en muestras chilenas con diferentes características. De la Barra evidenció cifras de MI entre $23 \%$ y $27 \%$ en población infanto-juvenil chilena ${ }^{23}$, datos que se aproximan a la estimación reportada en la literatura ${ }^{3}$, sobre una frecuencia de MI entre 2 a 3 veces mayor en pacientes con $\mathrm{TB}$ en comparación a la población no clínica. En relación a los subtipos de MI, es necesario remarcar la alta frecuencia de abuso sexual en nuestra muestra, cuantificada en $42 \%$ para ambos sexos, siendo una de las cifras más altas publicadas en pacientes bipolares ${ }^{24}$.

El estrés temprano podría conducir hacia un peor curso clínico de la enfermedad a través de diferentes mecanismos. El trauma temprano ha mostrado asociación con alteraciones en varios parámetros inflamatorios, como elevación de citoquinas pro-inflamatorias y proteína $\mathrm{C}$ reactiva $^{25,26}$. Dichas alteraciones permanecen incluso varios años después de la exposición al estresor, dando lugar a la hipótesis de una "huella" infla- 
matoria a lo largo del tiempo ${ }^{27}$. Las alteraciones en los procesos de neuroplasticidad también han sido vinculadas a antecedentes de trauma temprano. Pacientes bipolares con antecedente de MI presentan una disminución de niveles séricos de BDNF (factor neurotrófico derivado del cerebro) al compararlos con pacientes bipolares sin antecedentes de maltrato durante la infancia ${ }^{28}$. Las alteraciones en el funcionamiento del eje hipotálamo-hipófisis-adrenal (HHA) en pacientes víctimas de maltrato infantil también podrían conducir hacia una peor evolución clínica. Un interesante meta-análisis acerca de las alteraciones del eje HHA en el TB, concluye que tales alteraciones no presentarían características endofenotípicas, sino más bien estarían vinculadas a contextos de $\mathrm{MI}^{29}$. Desde un nivel conductual, el MI podría conducir a peores indicadores clínicos a través de dimensiones intermedias como los procesos de regulación emocional o de funcionamiento cognitivo ${ }^{30}$. Al respecto, nuestro estudio evidenció alteraciones en cognición social ${ }^{12,31,32}$, específicamente en pruebas de reconocimiento de emociones, aportando datos que contribuyen a la comprensión de la asociación entre MI , alteraciones en cognición social y un peor grado de funcionalidad y calidad de vida en pacientes con trastornos psiquiátricos ${ }^{33}$.

Pese a que la evidencia ofrece datos coincidentes en torno al rol del MI en el peor curso clínico del TB, las principales guías de recomendaciones de práctica clínica, así como los diseños de investigación, no han considerado tal variable con suficiente énfasis. La consistente evidencia sobre el rol de las experiencias adversas tempranas en el desarrollo de peores indicadores de salud en diferentes dominios ${ }^{34}$ obliga a la reflexión no sólo acerca de la consideración de nuevas hipótesis de causalidad en los Trastornos Mentales, sino también sobre la generación de nuevas aproximaciones terapéuticas. La alta frecuencia de MI en pacientes bipolares exige la promoción de nuevas estrategias de intervención psicosocial, que complementen los ya conocidos programas de psicoeducación propuestos por la guía GES-Minsal para el tratamiento del TB.

La reflexión que emerge del presente estudio propone además comprender la psicopatología del TB como un fenómeno dinámico y dependiente de la interacción con variables ambientales. Dicha interacción es la que estaría dando forma al conjunto de signos y síntomas observados, muy lejos de la idea de síntoma mental como expresión que emerge de forma pura a partir de una disfunción "interna, estática e inherente a la enfermedad". Nuestros datos plantean un desafío ético-clínico de carácter urgente para el tratamiento integral de pacientes con Trastorno Bipolar.

Agradecimientos: A los estudiantes de V año del departamento de psicología Clínica de la Universidad de Valparaíso quienes participaron activamente en diferentes etapas del proyecto de investigación.

\section{Referencias}

1. Read J, Sampson M, Critchley C. Are mental health services getting better at responding to abuse, assault and neglect? Acta Psychiatr Scand 2016; 134: 287-94.

2. WHO Library cataloguing in publication data. (2006). World Health Organization and international Society for Prevention of Child Abuse and Neglect. Preventing child maltreatment: a guide to taking action and generating evidence. Recuperado de: https://apps.who.int/ iris/bitstream/handle/10665/43499/9241594365_eng. pdf;jsessionid=1A31FF99764F07CA0B8CDB7CD9EE26FA? sequence $=1$.

3. Varese F, Smeets F, Drukker M, Lieverse R, Lataster T, Viechtbauer W, et al. Childhood adversities increase the risk of psychosis: a meta-analysis of patient-control, prospective- and cross-sectional cohort studies. Schizophr Bull 2012; 38 (4): 661-7.

4. Garno J, Goldberg J, Ramírez P, Ritzler B. Impact of childhood abuse on the clinical course of bipolar disorder. Br J Psychiatry 2005; 186: 121-5.

5. Daruy L, Brietzke E, Lafer B, Grassi-Oliveira R. Childhood maltreatment and clinical outcomes of bipolar disorder. Acta Psychiatry Scand 2011; 124 (6): 427-4.

6. Agnew-Blais J, Danese A. Childhood maltreatment and unfavourable clinical outcomes in bipolar disorder: a systematic review and meta-analysis. Lancet Psychiatry 2016; 3 (4): 342-9.

7. Etain B, Lajnef M, Brichant-Petitjean C, Geoffroy PA, Henry C, Gard S, et al. Childhood trauma and mixed episodes are associated with poor response to lithium in bipolar disorders. Acta Psychiatr Scand 2017; 135: 319-7.

8. Leverich G, Post R. Course of bipolar illness after history of childhood trauma. The Lancet 2006; 367 (9516): 1040-2.

9. Noto M, Noto C, Caribe A, Miranda-Scippa M, Nunez, 
S, Chavez A, et al. Clinical characteristics and influence of childhood trauma on the prodrome of bipolar disorder. Rev Bras Psiquiatr 2015; 37 (4): 280-8.

10. Bora E, Pantelis C. Social cognition in schizophrenia in comparison to bipolar disorder: a meta-analysis. Schizophr Res 2016; 175: 72-8.

11. Bora E. Neurocognitive features in clinical subgroups of bipolar disorder: a meta-analysis, J Affect Disord 2018; 15 (229): 125-34.

12. Samame C. Social cognition throughout the three phases of bipolar disorder: A state-of-the-art overview. Psychiatry Research 2013; 210: 1275-86.

13. Aas M, Dazzan P, Fisher H, Morgan C, Reichenberg A, Zanelli J, et al. Childhood trauma and cognitive function in first-episode affective and non-affective psychosis. Schizophr Res 2011; 129 (1): 9-12.

14. Spann MN, Mayes LC, Kalmar J, Guiney J, Womer F, Pittman B et al. Childhood abuse and neglect and cognitive flexibility in adolescents. Child Neuropsychol 2012; 182 (2): 182-9.

15. Pechtel P, Pizzagalli D. Effects of Early Life Stress on Cognitive and Affective Function: An Integrated Review of Human Literature. Psychopharmacology (Berl) 2011; 214 (1): 55-70.

16. Bernstein DP, Fink L, Handelsman L, Foote J, Lovejoy $\mathrm{M}$, Wenzel $\mathrm{K}$, et al. Initial reliability and validity of a new retrospective measure of child abuse and neglect. Am J Psychiatry 1994; 151: 1132-6.

17. Dominguez A, Behn A. Validación inicial del "Childhood Trauma Questionnaire-Short Form” versión española en Chile. Tesis (Magíster en Psicología Clínica) Pontificia Universidad Católica de Chile, 2018. https:// repositorio.uc.cl/handle/11534/22107.

18. McDonald S, Flanagan S, Rollins J, Kinch J.TASIT: A new clinical tool for assessing social perception after traumatic brain injury. Journal of Head Trauma and Rehabilitation 2003; 18: 219-38.

19. Carrasco M, Delgado B, Barbero M, Holgado F, del Barrio M. Psychometric properties of the Interpersonal Reactivity Index in Spanish child and adolescent population Psicothema 2011; 23 (4): 824-31.

20. Singer T, Klimecki O. Empathy and compassion. Curr. Biol 2014; 24: 875-8.

21. Muñoz-Neira C, Henríquez ChF, Ihnen JJ, Sánchez CM, Flores MP, Slachevsky ChA. Psychometric properties and diagnostic usefulness of the Addenbrooke's Cognitive Examination-Revised in a Chilean elderly sample. Rev Med Chile 2012; 140 (8): 1006-13.

22. Daruy L, Brietzke E, Lafer B, Grassi-Oliveira R. Child- hood maltreatment and clinical outcomes of bipolar disorder. Acta Psychiatry Scand 2011; 124 (6): 427-34.

23. De la Barra F, Rodríguez J, Álvarez P, Vergara A, Toledo V. Maltrato infantil y juvenil en una comuna Santiago. Parte I: estimación de la prevalencia. Rev Chil Pediatr 69 (3): 126-31 [citado 2019-07-07].

24. Maniglio R. Prevalence of child sexual abuse among adults and youths with bipolar disorder: a systematic review. Clin Psychol Rev 2013; 33 (4): 561-73.

25. Baumeister D, Akhtar R, Ciufolini S, Pariante C, Mondelli V. Childhood trauma and adulthood inflammation: a meta-analysis of peripheral C-reactive protein, interleukin-6 and tumour necrosis factor-. Mol Psychiatry 2016; 21 (5): 642-9.

26. Tursich M, Neufeld RW, Frewen PA, Harricharan S, Kibler JL, Rhind SG, et al. Association of trauma exposure with proinflammatory activity: a transdiagnostic meta-analysis Transl Psychiatry 2014; 22 (4): e413.

27) Slopen N, Kubzansky L, McLaughlin K, Koenen K. Childhood adversity and inflammatory processes in youth: a prospective study. Psychoneuroendocrinology 2013; 38 (2): 188-200.

28. Kauer-Sant'Anna M, Tramontina J, Andreazza A, Cereser K, da Costa S, Santin A, et al. Traumatic life events in bipolar disorder: impact on BDNF levels and psychopathology. Bipolar Disord 2007; 9 (1): 128-35.

29. Belvederi M, Prestia D, Mondelli V, Pariante C, Patti $\mathrm{S}$, Olivieri B, et al. The HPA axis in bipolar disorder: Systematic review and meta-analysis. Psychoneuroendocrinology 2016; 63: 327-42.

30. Aas M, Henry C, Andreassen OA, Bellivier F, Melle I, Etain B. The role of childhood trauma in bipolar disorders. Int J Bipolar Disord 2016; 4 (1): 2.

31. Frith CD. Social cognition. Philos Trans R Soc Lond B Biol Sci 2008; 12: 363 (1499): 2033-9.

32. Green M, Penn D, Bentall R, Carpenter W, Gaebel W, Gur R, et al. Social cognition in schizophrenia: an NIMH workshop on definitions, assessment, and research opportunities. Schizophrenia Bulletin 2008; 34 : 1211-20.

33. Fett A, Viechtbauer W, Domínguez M, Penn D, van Os J, Krabbendam L. The relationship between neurocognition and social cognition with functional outcomes in schizophrenia: a meta-analysis. Neurosci Biobehav Rev. 2011; 35 (3): 573-88.

34. Hughes K, Bellis M, Hardcastle K, Sethi D, Butchart A, Mikton C, et al. The effect of multiple adverse childhood experiences on health: a systematic review and meta-analysis. Lancet Public Health 2017; 2: 356-66. 